\title{
Laboratoire d'Essai à très Haute Tension.
}

\author{
Par J. VALLET, Ingénieur I. E. G.
}

Au moment où l'opinion publique se passionne à juste titre pour l'utilisation complete de nos forces hydrauliques et de la répartition de l'énergie ainsi produite sur l'ensemble du terriloire, il nous a paru intéressant de signaler les efforts faits par une importante Firme Lyonnaise, pour résoutre les nombreuses difficultés d'ordre technique que ce projet d'électrification soulèvera.

Si l'installation de grandes centrales hydrauliques ou thermiques ne crée point de problèmes absolument nouveaux, il n'en est pas de même du transport de l'énergie considérable ainsì engendrée à des distances dépassant 500 kilomètres, soit plus du double des distances actuelles.

Ces transports ne pourront être faits d'une façon économique et avec un rendement convenable que par l'emploi de très hautes tensions.

Quelques rares installations à 110.000 volts ont été réalisées sur le continent. En Amerique, des tensions plus élevées sont déjà employées. La tension qui sera adoptée en France pour le réseau de distribution générale, dont les études sont en cours, sera vraisemblablement celle de 120.000 volts. Pour certaines lignes spéciales, il est mème question de 200.000 ou 220.000 volts. Nous sommes loin des tensions actuellement utilisées en France et les constructeurs Français se trouveront en face de problèmes présentant des difficultés d'exécution nouvelles pour eux.

Il importe, et cela est capital, que ces réseaux à très haute tension, qui alimenteront nos principaux centres industriels, présentent la plus grande sûreté de fonctionnement en même temps qu'une sécurité absolue pour le personnel. Ces conditions ne pourront être remplies qu'à la suite d'essais extrêmement sérieux faits en usine pour éliminer toute pièce défectueuse dans le matériel utilisé : transformateurs, appareillage, isolateurs, câbles souterrains, etc.

Ces essais nécessiteront des laboratoires judicieusement installés et perriettant d'obtenir des tensions extrêmement élevées, supéreures a plus de deux fois la tension normale de service afin de se conformer aux règles d'essais que la pratique a reconnues nécessaires. Quelques chiffres permettront de mieux préciser notre pensée : Pour un transport a 120.000 volts, par exemple, les transformateurs devront être essayés à 240.000 volts, les isolateurs de la ligne à 250.000 volts, ces chiffres devront être à peu près doublés s'il s'agit d'une ligne à 220.000 volts.

Bien entendu, les tensions de claquage très intéressantes à connaitre pour le constructeur sont bien supérieures à celles-ci. Elles correspondent environ pour un transformateur à trois fois la tension normale, pour un isolateur à quatre et cinq fois cette même tension.

Le laboratoire spécial d'essais que viennent de réaliser les Ateliers de Constrictions électriques de Lyon é du Dauphiné permet d'obtenir la tension de 350.000 volts au moyen d'un transformateur monophasé, d'une puissance de 300 KVA, dont l'une des extrémités de l'enroulement haute tension est reliée à la borne, l'autre extrémité élant relićc à la masse. Il a été prévu l'adjonction d'un deuxième transformateur identique, monté en série avec le premier, ce qui permettra d'obtenir 700.000 volts entre les bornes extérieures des deux transformateurs.

Nous pensons intéresser le lecteur en lui donnant un bref apercu sur cette installation.

\section{TransformateUR 350.000 volts}

Ce qui trappe tout d'abord, ce sont les dimensions considérables du transformateur construit par les Aleliers de Constructions Flectriques de Lyon et du Dauphiné : $4 \mathrm{~m} .60$ de hauteur totale ; $2 \mathrm{~m} .50$ de diamètre, poids total en ordre de marche, 15 tonnes dont 5 tonnes d'huile $H$. T. de première qualité.

Il faut noter que la puissance mise en jeu est relativement consi- rable pour ce genre d'appareils. Il est, d'ailleurs, important que cetle puissance ne soit pas trop faible car les effluves, claquages et amorçages se produisent avec de petites puissances d'une façon différente qu'en service normal lorsque l'appareillage est placé sur un réseau de grande puissance, le moindre courant de surcharge provoquant immédiatement une forte chute de tension dans l'appareil.

On peut, d'ailleurs, déterminer d'une façon assez précise, la puissance nécessaire pour un transformateur d'essai par les considérations suivantes. Ces appareils étant destines a effectuer des essais d'isolement ne débitent normalement que sur des capacités (capacité entre enroulements et masse pour un transformateur, entre fil de ligne et terre pour un isolateur, entre conduct eur et terre pour un câble, etc... I'intensité du courant débité a pour valeur :

$$
\mathrm{I}=2 \pi f \mathrm{QU} 10^{-6}
$$

formule dans laquelle $C$ est la capacité en microfarads de l'appareil à essayer, U la tension d'essai et f la fréquence. La puissance en KVA du transformateur d'essai se dẻduit immédiatement :

$$
\mathrm{KVA}=2 \pi / \mathrm{CL}^{2} 10^{-4}
$$

formule qui montre en particulier que eette puissance doit croître comme le carré de la tension.

Pour certains essais de câbles armés, par exemple, la puissance nécessaire pent atteindre des valeurs assez considerables. Les exemples que nous donnons ci-dessous fixeront les idées à ce sujet. :

La capacité kilométrique d'un câble armé 60.000 volts monophasé d'une section de $100 \mathrm{~m} / \mathrm{m}^{2}$ est de 0,17 microlarads environ: Ce cable doit être normalement essaye à deux fois sa tension normale plus 40.000 volts, soit 160.000 volts pendant trois minutes et à sa tension normale plus 40.000 volts pendant trente minutes. En appliquant la formule ci-dessus, on trouve que le premier essai à 50 périodes exige une puissance de $250 \mathrm{KVA}$ pour une longueur rle câble de 200 mètres et le 2 essai une puissance de $100 \mathrm{KVA}$ pour la même longueur.

Un câble armé triphasć, 25.000 volts d'une section de $3 \times 120 \mathrm{~m} / \mathrm{m}^{2}$ possède une capacité kílométrique de 0,19 microfarad entre un conducteur et les deux autres mis à la terre. La tension d'essai est de 90.000 volts pendant trois minutes et 65.000 pendant 30 minu tes, ce qui correspond à une puissance de $150 \mathrm{KVA}$ pour une longueur de 300 mètres de câble pour le premier essai et $75 \mathrm{KVA}$ pour le deuxième.

Enfin, la capacité d'isolateurs pour 70.000 volts variable dans des limites assez larges suivant les constructeurs atteint parfois $\frac{1}{10^{4}}$ microfarad.

La tension d'essai est égale à deux fois la tension normale plus 10.000 volts soit 150.000 volts pendant 30 minutes.

L'essai de 50 isolateurs à cette tension et à la frécuence de 50 périodes nécessite une puissance de $40 \mathrm{KVA}$ environ.

Ces divers exemples montrent suffisamment la nécessité d'avoir un transformateur dont la puissance soit largement prévue lorsqu'on veut effectuer des essais d'appareils ayant une grande capacité et dont la construction soit très robuste pour pouvoir résister aux efforts assez considérables produits par un claquage toujours possible de l'appareil essayé.

Il faut ajouter que dans ce transformateur lisolement de l'enroulement haute tension et de la borne de sortie par rapport à la masse 
doit être dimensionné pour résister à la tension de 350.000 volts, alors que dans la plupart des transformateurs d'essai, le milien de lenroulement étant mis à la masse, l'isolement n'est prévu que pour la demi tension nominale du transformateur.

Cet isolement a été prévu très largement tant au point de vue claquage, que cheminement et effectué avee des isolants de première qualité ne travaillant, ainsi que l'huile, qu'à des contraintes admissibles.

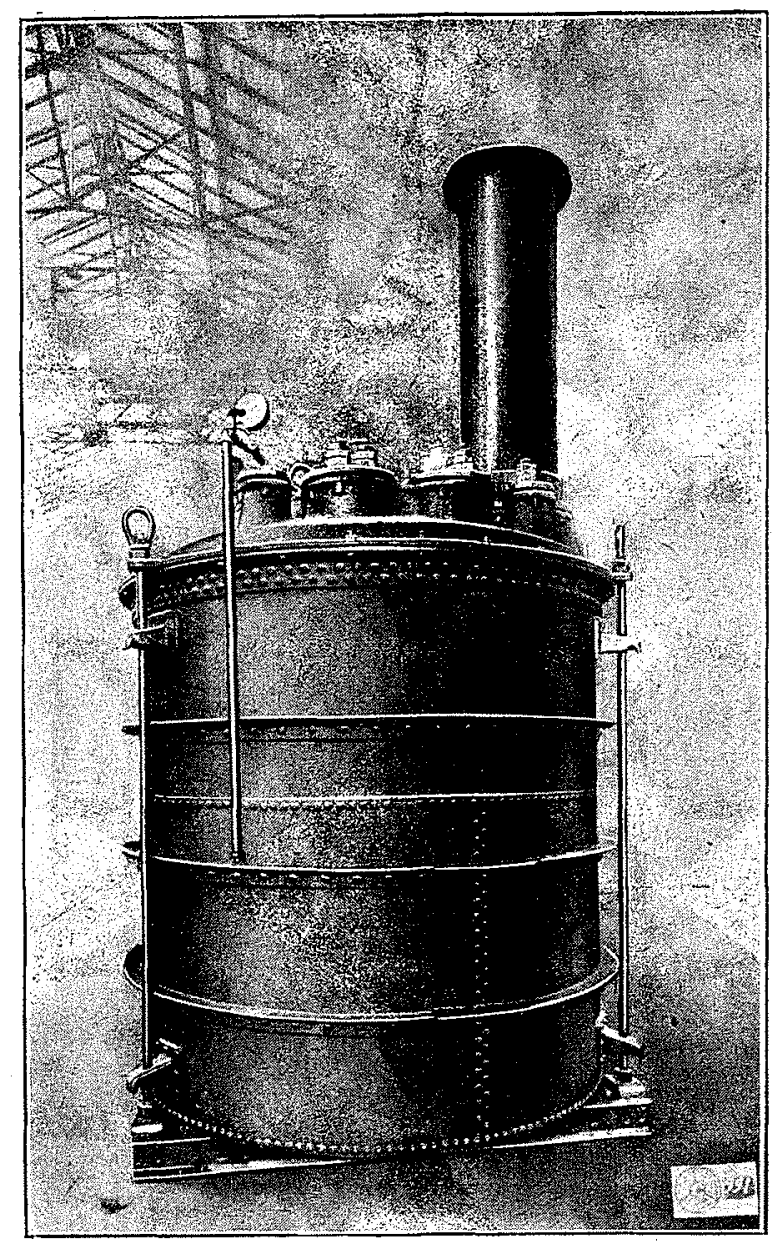

Vue du transformateur d'essai 350.000 volts $-300 \mathrm{KVA}$.

On est arrivé à ce résultat en répartissant judicieusement le travail du diélectrique isolant-huile. La construction a été conçue de façon à éviter toute concentration de potentiel par des arètes vives ou des angles et à donner une répartition de potentiel nettement prédéterminable.

L'isolement des galettes d'entrée a été réalisé d'une facon tròs méthodique et très large de manière à pouvoir résister à des tensions bien supérieures à celles prévues (galettes de choc). Un dispositif spécial permet d'assurer une égale répartition du potentiel sur toutes les spires de plusieurs galettes d'entrée. On remédie ainsi aux surélévations énormes de tension se produisant toujours sur les premières spires de l'enroulement.

En outre, les galettes de choc sont suivies d'autres galettes dans lesquelles l'isolement a été augmenté (galettes de demi-choc, galettes renforcées).

La borne de sortie du transformateur mérite une attention spéciale. Cette borne a été prévue pour 400.000 volts. Elle est du type condensateur o et a une longueur totale de $2 \mathrm{~m}$. 70 dont les deux tiers environ sont dans l'air, distance largement suffisante pour la tension en jeu. Elle se compose d'un certain nombre de cylindres concentriques séparés les uns des autres par une couche d'huile et comprenant chacun d'eux un tube métallique noyé dans un tube en hafelyte. Tous ces condensateurs sont de capacité identique et se répartissent également la tension de 400.000 volts. Le nombre des condensateurs et les épaisseurs des diélectriques ont ćté calculés pour que ces demiers ne soient soumis qu'À des contraintes acceptables.

Signalons que l'étanchéité est parfaite entre la cuve et son cou-

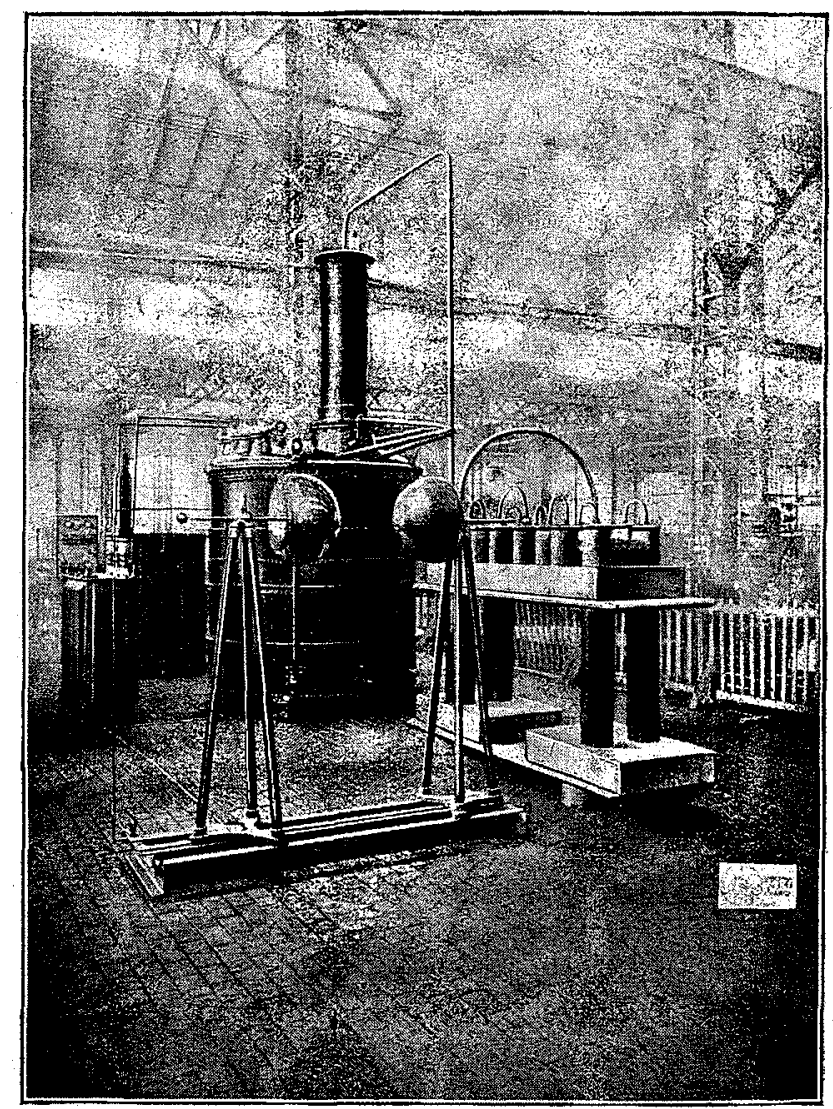

Vue montrant l'installation provisoire du transformateur d'essa avec les résistances, l'éclateur, le transformateur allxiliaire.

(Au $2^{\mathrm{e}}$ plan transformateur d'essai à 150.000 volts.)

vercle, de sorte que le transformateur et sa borne haute tension sont completement remplis d'huile, la borne servant de conservateur pour le bac. On évite ainsi à l'huile le contact cle l'air, condition essentielle pour lui conserver toutes ses qualités isolantes, qualités qui diminuent rapidement par l'oxydation et surtout par la moindre trace d'humidité.

Nous terminons cette rapide description en donnant queleques indications sur le circuit primaire. Ce dernier est prévu pour être alimenté à 5.000 volts et comporte quatre enroulements identiques dont les extrémités sont sorties sur le couvercle. Suivant le collplage adopté : parallèle, série-parallèle ou série, le rapport de transformation est $5.000 / 350.000-5.000 / 175.000$ ou $5.000 / 87.500$. Dans Ies deux derniers cas, la puissance du transformateur est réduite proportionnellement.

Le choix de la tension primaire à 5.000 volts a permis, malgré la puissance assez considćrable en jeu, d'utiliser une section de conducteur relativement réduite et de faciliter ainsi l'isolement des galettes et des prises par rapport à la haute tension. 


\section{Alimentation DU TRANSFORMATEUR D'ESSAI}

Un transformateur auxiliaire $200 / 5.000$ volts alimenté par le courant basse tension de l'usine est nécessaire et sa puissance a été choisie sensiblement égale à celle du transformateur à haute tension. Pour une installation de laboratoire d'essais, la détermination de cette puissance doit tenir compte de la durée des essais à effectuer. Pour des essais de càbles armés, d'isolateurs par exemple qui demandent parfois une marche presque ininterrompue pendant plusieurs heures, la puissance du transformateur d'alimentation sera égale à celle du transformateur d'essai.. Elle lui sera notablement inférieure s'il s'agit d'un fonctionnement intermittent de tres courte durée.

Ce transformateur auxiliaire est, lui-même, alimenté par l'intermédiaire d'un régulateur d'induction monophasé dans l'uir gui permet d'obtenir une variation continue de la tension d'alimentation et, par conséçuent, de la tension d'essai.

\section{Mesure des Tensions d’épreuve}

La mesure de tensions aussi élevées se fait par éclateur. I,éclateur employé est constitué par des sphères de $500 \mathrm{~m} / \mathrm{m}$ de diamètre. Ce genre d'éclateur doit toujours être utilisé à l'exclusion de l'éclateur à aiguilles pour les tensions supérieures à 50.000 volts.

Le montage de l'éclateur doit être établi en tenant compte d'un certain nombre de précautions pour éviter notamment que les sphères ne soient soumises à l'influence de champs électriques extérieurs.

Le réglage de la distance de l'éclateur sera différent selon que lappareil à essayer a une forte capacité ou, au contraire, une faible capacité. Il devra, en outre, tenir compte de la pression atmosphérique et de la température ; cette correction peut être considérable aux hautes altitudes. A titre d'indication, la distance d'éclatement à $25^{\circ}$ Centigrades et $760 \mathrm{~m} / \mathrm{m}$ de pression barométrique pour 350.000 volts avec des sphères de $500 \mathrm{~m} / \mathrm{m}$ dont l'une est à la terre, est de $224 \mathrm{~m} / \mathrm{m}$.

Il peut être utile cependant de brancher un voltmètre sur le circilit à 220 volts qui permettra un contrôle de la tension obtenue et ce suivre ses variations pendant l'essai.

Afin d'éviter les surtensions qui pourraient être provoquées prar les décharges des éclateurs, une résistance non inductive doit être insérée en série avec l'éclateur. Cette résistance, dont la valeur est apjroximativement de $1 \mathrm{ohm}$ par volt, est constituée par une série de culindres en carborandum (1). Elle amortit les oscillations des lautes fréquences au noment des éclatements et limite le courant à une valeur admissible. Il faut, d'ailleurs, ajouter que la tension de réactance du transformateur relativement élevée (environ $15 \%$ ) agit dans le meme sens êt le courant de court-circuit ne peut atteindre une valeur dangereuse. Cette disposition est préférable à celle qui consiste à établir Jes lignes à haute tension avec des fils fins pour utiliser l'effet de protection par effet "corona " sur ces fils.

\section{INSTALLATION D'Un LABORATOIRE D'ESSAI A TRÈS HAUTE TENSION}

Nous venons d'examiner les principaux éléments constitutifs d'un tel laboratoire. Nous insisterons beaucoup moins sur l'installation elle-même qui sera évidemment variable suivant la nature des essais à effectuer, ainsi que sur tous les appareils accessoires de mesure et de commande qui se retrouvent dans tous les lahoratoires.

l.es dispositifs de protection et de sécurité ont été particulièrement bien ćtudiés. Le laboratoire sera complètement entouré d'un grillage avec clispositif de verrouillage pour le rendre inaccessible lorsque la tension élevée est appliquée. La distance minima entre les murs, charpentes, etc... et les conducteurs sous tension est de 3 mètres. Ces derniers sont constitués par des tubes dont le diamètre est de $10 \mathrm{~m} / \mathrm{m}$ et ne présentent aucun coude bruscue.

Nous avons déjà vu que la protection était assurée par l'emploi cie résistances et par la forte tension de réactance du transformateur; de plus, un disjoncteur à huile sur le côté, 5.000 volts avec commánde automatique de divers points du laboratoire permettra en cas de langer de mettre le transformateur hors circuit.

I.es laboratoires de cette importance, tant au point re vue de la tension obtenue que de la puissance mise en jeu, sont actuellement assez rares. En dehors de leur utilité pour effectuer tous les essais que nous avons mentionnés précédemment, nous estimons qu'un laboratoire de ce genre permettra de pousser plus loin les recherches scientiljques et de contrôler par l'expérience les résultats obtenus par le calcul dans le domaine des très hates tensions. Bien des questions, notamment sur le champ électrique, effluves, eflet corona, contraintes diélectriques, répartition de potentiel, etc... gagneront à ctre précisées pour toutes les applications futures qui semblent levoir être très nombreuses.

(1) Provisoirement l'installation a été réalisée avec des résistances à liquide. 